\title{
Análisis de la contribución segmentaria en el rendimiento de las salidas de velocidad \\ Analysis of the segmental contribution in sprint start performance
}

\author{
*Marcos Gutiérrez-Dávila, *Carmen Gutiérrez-Cruz, **Jose Campos-Granell \\ * Universidad de Granada (España), **Universidad de Valencia (España)
}

\begin{abstract}
Resumen. En este estudio se ha aplicado un sistema de cálculo para examinar la contribución de cinco grupos de segmentos corporales al desplazamiento horizontal del CM en las salidas de tacos y explorar su relación con la velocidad horizontal del $\mathrm{CM}$ al final de $0.1 \mathrm{~s}$ y $0.312 \mathrm{~s}$ de la fase de aceleración. Han participado 20 velocistas con récord personal en $100 \mathrm{~m}$ de $11,05 \pm 0,31 \mathrm{~s}$. Se registraron las fuerzas horizontales de reacción mediante dos plataformas de fuerzas sincronizadas a una cámara de vídeo que registraba el plano sagital del movimiento. La contribución de las piernas al final de la fase de aceleración fue del 91.2 $\pm 2.4 \%$ y la pierna libre del $8.1 \pm 1.0 \%$. El tronco + cabeza es el primer grupo de segmentos que comienza a contribuir al desplazamiento horizontal del CM, alcanzando el $39.3 \pm 24.3 \%$ en los primeros $0.1 \mathrm{~s}$. Su rápida contribución hacia delante provoca una fuerza reactiva hacia atrás que podría estar relacionada con una dorsiflexión de los tobillos de $8^{\circ} \pm 3^{\circ}$ y $9^{\circ} \pm 3^{\circ}$, pierna retrasada y adelantada, respectivamente. La contribución temprana de la cabeza +tronco se ha correlacionado positivamente con la velocidad horizontal al final de la fase de aceleración ( $\mathrm{r}=0.622, \mathrm{p}=0.003)$, lo que confirma su importancia como indicador de rendimiento en las salidas de tacos.
\end{abstract}

Palabras clave: Biomecánica, Cálculo de medidas, Técnica de salida, Atletismo.

\begin{abstract}
In this study, a calculation system has been applied to examine the contribution of five groups of body segments to the horizontal displacement of the Center of Mass (CM) in block starts and to explore their relationship with the horizontal velocity of the $\mathrm{CM}$ at the end of $0.1 \mathrm{~s}$ and $0.312 \mathrm{~s}$ of the acceleration phase. Twenty sprinters with a personal record in $100 \mathrm{~m}$ of $11.05 \pm 0.31 \mathrm{~s}$ have participated. The horizontal reaction forces were recorded using two force platforms synchronized to a video camera that recorded the sagittal plane of motion. The contribution of the legs at the end of the acceleration phase was $91.2 \pm 2.4 \%$ and the free leg was $8.1 \pm 1.0 \%$. The trunk + head is the first group of segments that begins to contribute to the horizontal displacement of the CM, reaching $39.3 \pm 24.3 \%$ in the first $0.1 \mathrm{~s}$. His quick forward contribution causes a reactive backward force that could be related to an ankle dorsiflexion of $8^{\circ} \pm 3^{\circ}$ and $9^{\circ} \pm 3^{\circ}$, back and front leg, respectively. The early contribution of the head + trunk has been positively correlated with the horizontal velocity at the end of the acceleration phase $(\mathrm{r}=0.622, \mathrm{p}=0.003)$, which confirms its importance as a performance indicator in block starts.
\end{abstract}

Key words: Biomechanics, Measurement calculation, Sprint Start technique, Athletics.

\section{Introducción}

El rendimiento en la salida de tacos es un factor decisivo en las carreras de 60 y 100 metros, llegándose a obtener alrededor del 30\% de la máxima velocidad en un tiempo inferior a 0.4 s. (Slawinski et al., 2010, Bezodis, Salo \& Trewartha, 2010). Además de haberse documentado como la fase más crítica del rendimiento de estas pruebas (Willwacher, et al., 2016), su relevancia también se puede constatar por la cantidad de publicaciones que han tratado de dar explicación a los mecanismos que condicionan su rendimiento (Bezodis, Willwacher \& Salo, 2019; Harland \& Steele, 1997).

Fecha recepción: 31-05-21. Fecha de aceptación: 21-09-21

Carmen Gutiérrez-Cruz

carmengc90@hotmail.com
En la mayoría de estas publicaciones se han utilizado tacos instrumentalizados para registrar las fuerzas externas y obtener, a partir de ellas, las variables de rendimiento asociadas al CM (Bezodis, Walton \& Nagahara, 2019; Gutiérrez-Dávila, Dapena \& Campos, 2006; Otsuka, et al., 2014). En ocasiones también se han utilizado sistemas de análisis del movimiento que han permitido obtener la cinemática y cinética articular de los miembros inferiores (Bezodis et al., 2015; Brazil et al., 2017; Schrödter, Brüggemann, Willwacher, 2017; Slawinski et al., 2010). Sin embargo, son escasas las publicaciones donde se ha estudiado la contribución de los segmentos corporales al rendimiento de las salidas de tacos. Una carencia que sorprende al considerar que las aceleraciones producidas al CM son directamente proporcionales al sumatorio del producto entre las masas de los segmentos corporales y sus respectivas aceleraciones. En este sentido, nos sumamos a las consideracio- 
nes expuestas por Brazil et al. (2018) cuando señalan que el conocimiento de la cinética articular de la parte superior del cuerpo podría ayudar a explicar la variación en el rendimiento de las salidas de tacos, una sugerencia que toma especial relevancia cuando se trata de segmentos corporales como el tronco que superan el 43\% de la masa corporal.

En relación a la contribución del tronco, es necesario considerar que, partiendo de una posición de «listos», donde las caderas están más elevadas que los hombros, la extensión inicial del tronco y la cabeza producirá una aceleración horizontal de su centro de masas $(\mathrm{cm})$ y, como reacción, una fuerza hacia atrás contra las piernas que incrementará la fuerza ejercida por los miembros inferiores contra los tacos. Un mecanismo que justificaría la importancia de cuantificar su contribución al desplazamiento horizontal del CM en el inicio de aplicación de fuerzas contra los tacos. Apoyando esta idea, son varias las publicaciones que sugieren la importancia de esta acción temprana del tronco al describir la existencia de un patrón próximo-distal de participación articular que comienza con la activad extensora de las articulaciones de las caderas y el desplazamiento angular del tronco (Bezodis et al., 2015; Brazil et al., 2017; Debaere, Delecluse, Aerenhouts, Hagman \& Jonkers, 2013; Slawinski, et al., 2010).

Son varias las aportaciones que han descrito un periodo de absorción de energía junto a la dorsiflexión de ambos tobillos antes de comenzar su flexión plantar definitiva, lo que ciertos autores han asociado a una actividad reactiva del ciclo de estiramiento acortamiento en el inicio de la salida de tacos (Brazil et al., 2017; Guissard, Duchateau \& Hainaut, 1992; Mero, Kuitunen, Harland, Kyröläinen \& Komi 2006). En este mismo sentido, Schrödter et al. (2017) han obtenido correlaciones positivas entre la amplitud y la máxima velocidad de estiramiento dorsiflexor de los tobillos con el impulso y la máxima fuerza de empuje horizontal, sugiriendo la importancia que tiene el ciclo estiramiento-acortamiento de la unidad músculo-tendinosa del sóleo en la generación de fuerza. Sin embargo, estos mismos autores no confirman la existencia de un ciclo estiramiento acortamiento en términos fisiológicos y sugieren el uso de otras tecnologías para confirmarlo. Mediante el análisis de la contribución segmentaria que se presenta en este estudio es posible cuantificar el desplazamiento horizontal de los miembros inferiores al transmitir la fuerza reactiva que provoca la extensión temprana del tronco, lo que podría ser parte del efecto causal de la dorsiflexión descrita en ambos tobillos.
El propósito principal de este estudio ha sido aplicar un novedoso sistema de cálculo para examinar la contribución de cinco grupos de segmentos corporales al desplazamiento horizontal del CM durante la aplicación de fuerzas contra los tacos. Como objetivo complementario, se pretende explorar su relación con la velocidad horizontal desarrollada durante la salida. A partir de las consideraciones expuestas, planteamos las siguientes hipótesis: a) la mayor contribución al desplazamiento horizontal del CM lo realizará la pierna más adelantada, b) El movimiento horizontal del CM se inicia con la contribución positiva del tronco más la cabeza, acompañada de un cierto retroceso de las piernas por efecto de la fuerza reactiva que produce la aceleración hacia delante del tronco y c) Tras el despegue de la pierna retrasada ésta mantiene una contribución horizontal al desplazamiento del CG hasta el despegue de la pierna adelantada. La exploración correlacional de las contribuciones segmentarias con la velocidad horizontal del CM confirmará la importancia de la aceleración del tronco hacia delante en el rendimiento de las salidas de tacos.

\section{Material y método}

\section{Participantes}

En este estudio han participado 20 velocistas masculinos, expertos en carreras de velocidad y en activo durante la toma de los datos (edad $=20,3 \pm$ 3,0 años; talla $=1,78 \pm 0,06 \mathrm{~m}$; masa $=71 \pm 6 \mathrm{~kg}$; récord personal en $100 \mathrm{~m}=11,05 \pm 0,31 \mathrm{~s})$. Se obtuvo el consentimiento informado de los participantes, siguiendo las directrices de la Comisión de Ética de la Universidad.

\section{Materiales y pruebas}

La recogida de datos tuvo lugar en el Palacio $\mathrm{Cu}$ bierto Luis Puig de Valencia, España, una semana antes de la Competición Nacional más importante de la temporada de pista cubierta. Se habilitó una superficie de tartán de 20 x1.20 metros para posibilitar el uso de calzado de clavos. Las dos plataformas de fuerza fueron implementadas con este mismo material.

El día anterior a la sesión de recolección de datos, los participantes practicaron salidas utilizando los tacos instrumentalizados para el estudio. Los tacos se ajustaron a una posición intermedia de distancia entre ellos $(0.350 \mathrm{~m})$ y una inclinación de $60^{\circ}$ con respecto a la horizontal. La distancia entre tacos se mantuvo fija para todos los participantes, aunque se les permitió apoyar las manos en el suelo donde consideraron que mantenían la posición más cómoda. Este mismo protocolo se 
utilizó para la toma de datos. Para familiarizarse con las órdenes de salida se utilizaron los mismos sistemas automáticos que los utilizados en la posterior de toma de datos. Este sistema automático de salida producía un sonido de tono bajo para representar la orden «listos» $\mathrm{y}$ un sonido de tono alto para indicar el disparo de salida. Para reducir un posible proceso de aprendizaje y anticipación, el tiempo entre los dos sonidos se varió aleatoriamente entre valores extremos de 1,5 s y 3,0 S.

Se instruyó a los participantes para que corrieran lo más rápido posible hasta una línea de meta situada a 10 $m$ del inicio. Con un breve descanso entre ensayos (entre 10 y 12 minutos), cada participante realizó salidas consecutivas hasta obtener cinco ensayos válidos. Las fuerzas de reacción del suelo se obtuvieron de dos plataformas de fuerza Dinascan / IBV (Instituto de Biomecánica de Valencia, Valencia, España) a una frecuencia de muestreo de $250 \mathrm{~Hz}$. La plataforma A (600 $\times 370 \mathrm{~mm}$ ) se colocó debajo de los tacos de salida y la plataforma B $(800 \times 800 \mathrm{~mm})$ se colocó debajo de las manos del participante.

Todos los ensayos se grabaron a $250 \mathrm{~Hz}$ con una cámara de video digital de alta velocidad Redlake MotionScope PCI 1000S (Redlake Corporation, San Diego, CA). La cámara de vídeo se situó perpendicular al plano sagital del velocista en posición de «listos» y a una altura de $0.6 \mathrm{~m}$. Antes de la grabación, se filmó un sistema de referencia, que consistía en un prisma rectangular de $2 \mathrm{~m} \times 2 \mathrm{~m} \times 0.5$, cuya proyección vertical de su centro geométrico coincidió con el centro de la plataforma B. La escala que permitió transformar las coordenadas en datos reales se estableció a partir del plano medio del prisma rectangular. Las dos plataformas de fuerza y la cámara de vídeo se sincronizaron a través de una señal electrónica producida por el sistema automático de salida.

\section{Recolección de los datos}

En cada ensayo se calcularon los valores medios de las fuerzas registradas en las dos plataformas durante un período de $0.08 \mathrm{~s}$ después de perder contacto el participante con las plataformas (20 muestras consecutivas). Para eliminar un posible error sistemático, estos valores de referencia se restaron a todas las demás lecturas de la plataforma de fuerza. El inicio de movimiento se determinó a partir de la fuera horizontal resultante de los registros de las dos plataformas de fuerzas. Se estimó en 0,002 s (medio intervalo) antes del instante en que la fuerza horizontal alcanzó por primera vez un va- lor igual o superior al 1\% del peso corporal. El final del apoyo de la mano se estimó en 0,002 s antes del primer instante en que la fuerza vertical de la plataforma $\mathrm{B}$ mostró un valor negativo. El final de la aplicación de fuerza contra los tacos (despegue del pie adelantado) se estimó en $0.002 \mathrm{~s}$ antes de que la fuerza horizontal de la plataforma A mostrara el primer valor negativo. Se asignaron valores cero a todas las fuerzas de la plataforma $\mathrm{B}$ después del despegue de las manos y a todas las fuerzas de la plataforma A después del despegue del apoyo retrasado. Esto se realizó para eliminar el ruido aleatorio de bajo nivel de las dos plataformas durante el tiempo en que no había contacto.

Para cada participante se seleccionó un ensayo entre los cinco registrados para el análisis completo de vídeo, utilizando como criterio el valor mediano de la velocidad horizontal del CM alcanzada en el despegue del pie adelantado, determinándose dichas velocidades a partir de los respectivos registros de las fuerzas horizontales, según procedimiento expuesto en Gutiérrez-Dávila et al. (2006). La estabilidad de los datos se cuantificó mediante el coeficiente de correlación intraclase aplicado a los cinco registros de la velocidad horizontal en el despegue para cada participante (ICC $=0.934)$.

Las imágenes de video se digitalizaron de forma manual a una frecuencia de $125 \mathrm{~Hz}$, utilizando para ello el software Cyborg 2.0 (Unisport-Junta de Andalucía, España). Se utilizó un modelo de 14 segmentos definido por 21 marcadores colocados en el cuerpo del velocista (extremos anterior y posterior de los pies; centros articulares de tobillos, rodillas, caderas, hombros, codos y muñecas; tercer metacarpiano de las manos; hueco supraesternal; ángulo del maxilar y vértex). Estos marcadores se posicionaron según el modelo y los parámetros inerciales propuestos por Zatsiorsky \& Seluyanov (1983) y adaptados por de Leva (1996). Las coordenadas de los 21 marcadores corporales se interpolaron a $250 \mathrm{~Hz}$, utilizando el método de splines elevados a la quinta potencia (Wood \& Jennings, 1979). Para evitar la posible introducción de errores sistemáticos, las funciones splines se aplicaron con nivel de suavizado cero.

\section{Cálculo de las medidas de rendimiento}

Las posiciones del CM para cada imagen se determinaron a partir del modelo y los parámetros inerciales propuestos por Zatsiorsky \& Seluyanov (1983) y adaptados por de Leva (1996). Para determinar la contribución segmentaria al desplazamiento horizontal del CM durante la salida de tacos, se han considerado cinco gru- 
pos de segmentos corporales: Brazo del taco posterior (BTP) y Brazo del taco anterior (BTA) corresponden a las respectivas masas del brazo antebrazo y mano; Tronco+Cabeza (TC) se considera la masa del tronco más la cabeza, incluido el cuello; Pierna libre (PL) hace referencia al miembro inferior (muslo, pierna y pie) del taco más retrasado (sólo se considera a partir del despegue del taco). Piernas apoyadas en tacos (PAT) son los miembros inferiores que están en contacto con los tacos (dos apoyos hasta el despegue del pie retrasado y un apoyo desde ese instante hasta el despegue del pie adelantado).

Para el cálculo de la contribución de cada grupo de segmentos al desplazamiento horizontal del CM del velocista, se han adaptado los procedimientos descritos por Gutiérrez-Dávila, Amaro, Garrido \& Rojas (2014) para el salto vertical, según las siguientes relaciones

$$
\begin{aligned}
& C_{(\overline{G T P}) i}=\Delta X_{\bar{B} P i} \cdot m_{\bar{B}} ; C_{(\bar{B} T A) i}=\Delta X_{\bar{B} A i} \cdot m_{\bar{B}} \\
& C_{(T C) i}=\left(\Delta X_{(T C+2 B) i^{*}} m_{(T C+2 \bar{B})}\right)-\left(C_{(\bar{B} T P)_{i} i}+C_{(\bar{B} T A) i}\right) \\
& C_{(P L) i}=\Delta X_{P L i} \cdot m_{P} \\
& C_{(P A T) i}=\Delta X_{C M i}-\left(C_{(B T P) i}+C_{(B T A) i}+C_{(T C) i}+C_{(P L) i}\right)
\end{aligned}
$$

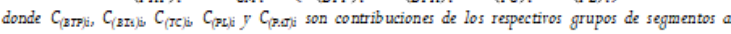
morimiento horizontal del CM para el interralo de tiempo i; $X_{R p}$ y $X_{\mathrm{R}}$ es la posición horizontal relatira del $\mathrm{cm}$ de los brazos al marcador supreesternal; $X_{\mathrm{TC}+3 \mathrm{~s}}$ es la posición horizontal relatira del $\mathrm{cm}$ de

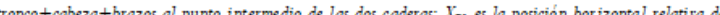

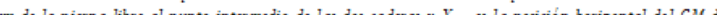

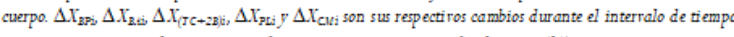
$i ; \mathrm{m}_{2}, \mathrm{~m}_{\mathrm{T}} \mathrm{TC}+2 \mathrm{l}, \mathrm{m}, \mathrm{m}$ son los porcentajes de sus respectiras masas a todo el cuerpo $(\%)$

A partir de las relaciones expuestas, la contribución de los respectivos grupos de segmentos para cada periodo temporal se calculó sumando las contribuciones consecutivas para cada intervalo. Todos los datos fueron normalizados con respecto a la longitud de piernas de los velocistas (distancia entre el suelo y el epicóndilo externo del fémur estando de pie). Para realizar las representaciones gráficas, los datos de todos los participantes se volvieron a muestrear utilizando una interpolación con splines elevados a la quinta potencia para obtener valores en porcentajes del tiempo total de la fase de aceleración (para cada participante el 100\% correspondía al tiempo de aplicación de fuerzas contra los tacos).

Para evaluar el rendimiento y facilitar las comparaciones se ha utilizado la velocidad horizontal del CM cuando se alcanzaban dos tiempos concretos de la fase de aceleración. Así, para la evaluación del rendimiento de la salida de tacos, el tiempo se truncó para todos los participantes en el menor de todos los registrados (0.312 s). Para evaluar con detalle el rendimiento durante la primera parte de la fase de aceleración, el tiempo se truncó para todos los participantes en el menor de los registrados en el instante de despegue de las manos (0.1 s).

Finalmente, para cada imagen se han obtenido los ángulos de las articulaciones de los dos tobillos, dos rodillas y las dos caderas, utilizando para ello el producto escalar de los vectores que identifican sus respectivos segmentos corporales. Para sus representaciones gráficas, los datos se interpolaron para obtener valores en porcentajes del tiempo total de la fase de aceleración, utilizando el mismo procedimiento que para la representación de las contribuciones de los distintos grupos de segmentos.

\section{Análisis estadístico}

Los datos descriptivos se presentan numéricamente como media \pm desviación estándar. Para todas las variables de interés se verificó la normalidad de los datos mediante una prueba de Shapiro-Wilk, obteniéndose en todas ellas una distribución normal ( $p$ osciló entre 0.435 y 0.759$)$. Se calcularon los coeficientes de correlación de Pearson entre la velocidad horizontal alcanzada por del CM en los dos rangos temporales de movimiento $(0.1 \mathrm{~s}$ y $0.312 \mathrm{~s}$ ) y las correspondientes contribuciones acumuladas por los distintos grupos de masas segmentarias. La significación estadística se aceptó con $P<0,05$. Para todo ello se ha utilizado el paquete estadístico SPSS (IBM SPSS versión 22.0, Chicago, IL, USA).

\section{Resultados}

En la figura 1(a) se ilustran los datos de tendencia central de la fuerza horizontal resultante frente al tiempo expresado en porcentaje de la fase de aceleración, constatándose que el pico máximo de fuerza horizontal se produce antes del despegue de las manos. A continuación, tiende a descender hasta el despegue del pie

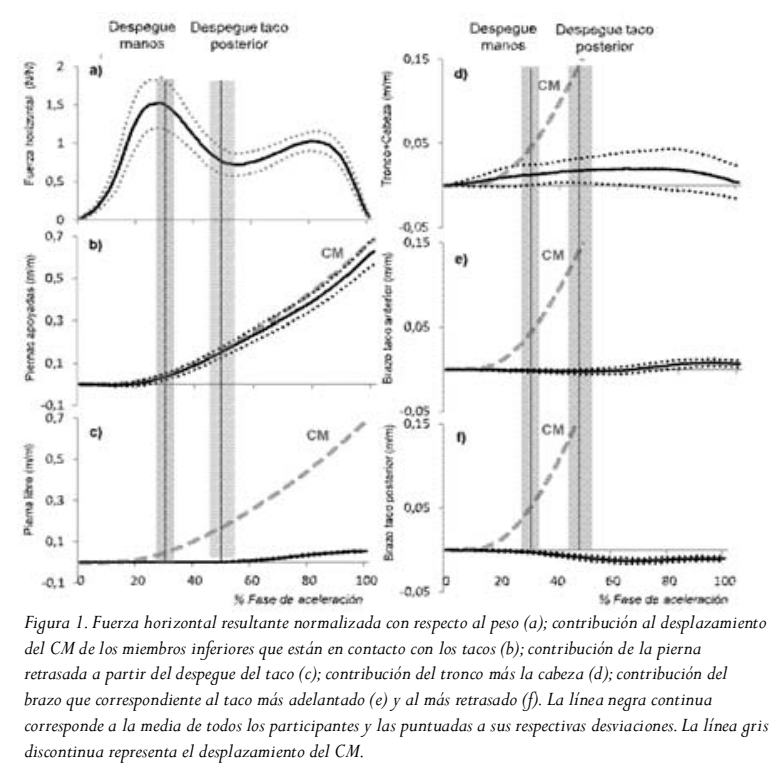


con el taco posterior, donde de nuevo vuelve a incrementarse hasta alcanzar un segundo pico cuando sólo empuja contra el taco anterior y la pierna libre se acelera hacia delante.

En la figura 1 también se ilustran las contribuciones de los distintos grupos de segmentos al desplazamiento del CM frente al tiempo expresado en porcentaje de la fase de aceleración. En la tabla 1 se presentan estos mismos datos en forma numérica y expresados en porcentajes del desplazamiento del CM para los primeros 0.1 s y 0.312 s de la fase de aceleración y en el instante del despegue del taco anterior. Como se esperaba, la mayor contribución al desplazamiento horizontal del CM al final de la fase de aceleración la realizan los miembros inferiores que están en contacto con los tacos

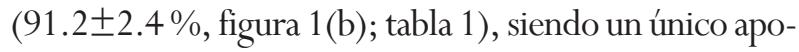
yo desde el despegue del taco posterior hasta el final de la fase de aceleración. Tras el despegue del taco posterior, la pierna libre sigue contribuyendo al desplazamiento horizontal del CM hasta el despegue del taco anterior ( $8.1 \pm 1.0 \%$, figura $1(\mathrm{c})$; tabla 1$)$. El tronco + cabeza es el primer grupo de segmentos que comienza a contribuir al desplazamiento horizontal hacia delante del CM, alcanzando una contribución de 39.3 $\pm 24.3 \%$ en los primeros $0.1 \mathrm{~s}$ de la fase de aceleración. Dicha contribución tiende a reducirse tras el despegue de las manos (ver figura 1(d), tabla 1). Los brazos tienden a desplazar el CM hacia atrás hasta el despegue del taco posterior (contribución negativa). A continuación, la contribución del brazo correspondiente al taco anterior comienza a tener una ligera contribución positiva, aunque con valores irrelevantes, mientras que el brazo que corresponde al taco posterior sigue manteniendo una cierta contribución negativa.

\begin{tabular}{|c|c|c|c|}
\hline Item & $\begin{array}{c}\text { Primeros } 0.1 \mathrm{~s} \mathrm{de} \\
\text { aceleración }\end{array}$ & $\begin{array}{c}\text { Primeros } 0.312 \mathrm{~s} \\
\text { de aceleración }\end{array}$ & $\begin{array}{c}\text { Despegue taco } \\
\text { anterior }\end{array}$ \\
\hline Desplazamiento horizontal $\mathrm{CM}(\mathrm{m} / \mathrm{m})$ & $0.037 \pm 0.019$ & $0.490 \pm 0.080$ & $0.673 \pm 0.055$ \\
\hline Contribución de apoyos en tacos $(\%)$ & $69.5 \pm 22.4$ & $89.3 \pm 4.3$ & $91.2 \pm 2.4$ \\
\hline Contribución de pierna libre (\%) & & $8.1 \pm 1.1$ & $8.1 \pm 1.0$ \\
\hline Contribución de tronco + cabeza ( $\%)$ & $39.3 \pm 24.3$ & $3.8 \pm 3.7$ & $1.2 \pm 2.1$ \\
\hline Contribución brazo taco anterior (\%) & $-3.3 \pm 3.4$ & $1.3 \pm 1.1$ & $0.0 \pm 0.0$ \\
\hline Contribución brazo taco posterior (\%) & $-5.0 \pm 4.1$ & $-2.3 \pm 1.1$ & $-1.4 \pm 0.8$ \\
\hline Velocidad horizontal del $\mathrm{CM}\left(\mathrm{ms}^{-1}\right)$ & $0.72 \pm 0.19$ & $2.82 \pm 0.34$ & $3.23 \pm 0.24$ \\
\hline
\end{tabular}

Las gráficas que se presentan en la figura 1 indican que existe una secuencia en la contribución al desplazamiento horizontal del CM, comenzando con el tronco + cabeza, seguido de los segmentos que están en contacto con los tacos y, a continuación, la pierna libre, mientras que la contribución de los brazos al desplazamiento hacia delante del CM es irrelevante. Para examinar con detalle la secuencia entre la contribución del tronco + cabeza y los segmentos que están en contacto con los tacos durante la primera parte de la fase de aceleración, en la figura 2 se ilustran las contribuciones de ambos grupos de segmentos y la contribución media de los dos brazos al desplazamiento horizontal del CM. Se observa que durante los primeros instantes $(\sim 18 \%$ de la fase de aceleración), el tronco + cabeza contribuyen al desplazamiento hacia delante del CM, mientras que las piernas lo hacen en sentido contrario (contribución negativa). El desplazamiento del CM hacia delante se produce por la mayor contribución positiva de la cabeza+tronco con respecto a las contribuciones negativas que mantienen las piernas y los brazos. A continuación, las piernas se suman a la contribución positiva del tronco + cabeza para alcanzar en el $23.7 \pm 4.2 \%$ de la fase da aceleración el pico máximo de fuerza.

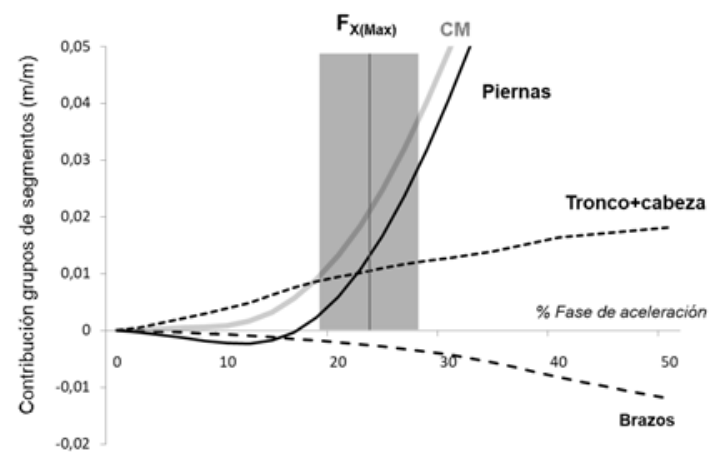

Figura 2. Contribución al desplazamiento del $C M$ de las piernas, troncotcabeza y la media de los dos brazos en la primera mitad del tiempo de la fase de aceleración. La línea gris representa el desplazamiento del CM. La línea vertical y espacio sombreado indican el porcentaje medio \pm la desviación estándar donde se produce el pico máximo de la fuerza horizontal.

En la figura 3 se presentan los datos de tendencia central para los ángulos de las articulaciones de las caderas, rodillas y tobillos de las dos piernas, frente al tiempo expresado en porcentaje de la fase de aceleración. Se observa una extensión inicial de las dos caderas y rodillas y una dorsiflexión de los dos tobillos previa la
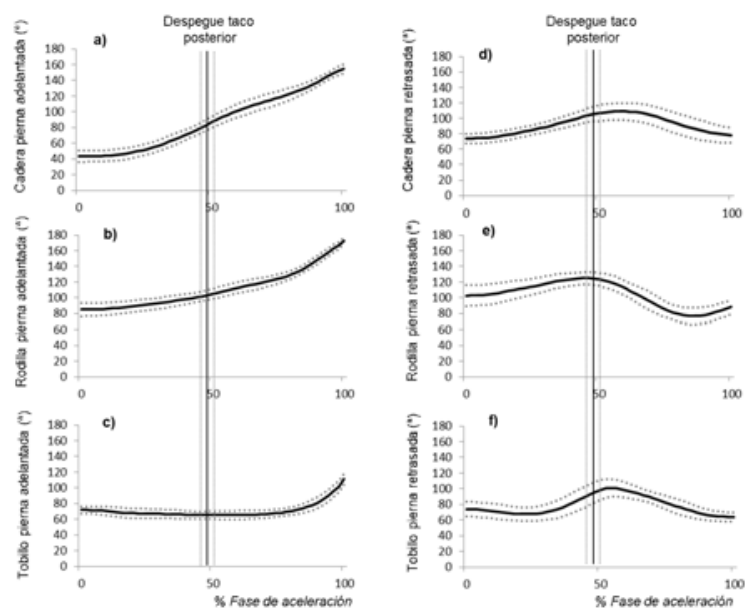

Figura 3. Media y desviación estándar de los ángulos las articulaciones de la cadera, rodilla y tobillo de lo pierna adelantada (a,b,c, respectivamente) y retrasada (d,e,f, respectivamente), frente al tiempo expresado en porcentajes de la fase de aceleración 
flexión plantar que se produce antes del despegue de sus respectivos tacos.

En la tabla 2 se presentan los coeficientes de correlación de Pearson entre la velocidad horizontal alcanzada por del CM en los dos rangos temporales de movimiento $(0.1 \mathrm{~s}$ y $0.312 \mathrm{~s})$ y las correspondientes contribuciones acumuladas por los grupos de masas segmentarias que han obtenido una cierta significación estadística. Solo se han obtenido valores de correlación significativos entre la contribución de las piernas de apoyo y la cabeza+tronco en el final de $0.1 \mathrm{~s}$ con la velocidad alcanzada por el $\mathrm{CM}$ al finalizar los dos rangos temporales de movimiento $(0.1 \mathrm{~s}$ y $0.312 \mathrm{~s})$. Para completar el análisis, se ha incluido la correlación positiva que ha existido entre la velocidad del $\mathrm{CM}$ al final de $0.1 \mathrm{~s}$ de la fase de aceleración y la alcanzada al final de $0.312 \mathrm{~s}(\boldsymbol{r}=0.764$, pÂ0.000).

Tabla 2.

Datos de correlaciones entre las velocidades alcanzadas por el CM al final de $0.1 \mathrm{~s}$ and $0.312 \mathrm{~s}$ de la fase de aceleración y las contribuciones de los grupos de segmentos que han alcanzado una significación superior al $95 \%$.

\begin{tabular}{lcccc}
\hline & \multicolumn{2}{c}{ Velocidad del CM } & \multicolumn{2}{c}{ Velocidad del CM en } \\
& \multicolumn{2}{c}{ en $0.1 \mathrm{~s}$} & \multicolumn{2}{c}{$0.312 \mathrm{~s}$} \\
& $r$ & $P$ & $r$ & $P$ \\
\hline Velocidad del CM al final de $0.1 \mathrm{~s}\left(\mathrm{~ms}^{-1}\right)$ & 1 & - & 0.764 & 0.000 \\
Contribución de las piernas en $0.1 \mathrm{~s}(\%)$ & 0.487 & 0.030 & 0.575 & 0.008 \\
Contribution del tronco+cabeza en $0.1 \mathrm{~s}(\%)$ & 0.467 & 0.038 & 0.622 & 0.003 \\
\hline
\end{tabular}

\section{Discusión}

Se constata la existencia de una secuencia próximodistal de contribución segmentaria al desplazamiento horizontal del CM. Comienza con la participación del tronco + cabeza, seguida de la contribución positiva de las piernas apoyadas en los tacos y de la pierna libre tras su despegue del taco posterior. La contribución de los brazos al desplazamiento hacia delante del CM ha sido insignificante debido a su escasa masa y a los distintos sentidos del movimiento horizontal que adquieren cada uno de ellos tras su despegue del suelo. Estos resultados son coincidentes con los aportados por otros investigadores a partir de la goniometría articular (Bezodiset al., 2015 Slawinski et al., 2010; Slawinski et al., 2013; Debaere et al., 2013; y Brazil, et al., 2017)

El rápido incremento inicial de la contribución horizontal del tronco + cabeza indica la presencia de una aceleración horizontal hacia delante que, debido a su gran masa, produce una considerable fuerza reactiva hacia atrás que debe ser transmitida a los tacos a través de las piernas que están en contacto con ellos, lo que favorece al primer pico de la fuerza de reacción horizontal (ver figura 1(a)). Así, las piernas reciben momentáneamente una fuerza horizontal compresiva que provocará un cierto desplazamiento hacia atrás del CM (contribución negativa), a la que se suma la contribución negativa de los dos brazos. En esta situación, para conseguir un cierto desplazamiento horizontal hacia delante del CM, las contribuciones negativas de las piernas y los brazos tienen que ser compensadas y superadas por la mayor contribución positiva (hacia delante) del tronco + cabeza (ver figura 2). La contribución negativa provocada por las piernas, podría estar relacionada con la dorsiflexión de los tobillos y/o flexión de las rodillas. Los datos registrados para los ángulos de estas articulaciones durante los primeros instantes de la fase de aceleración, indican que existe una dorsiflexión en los dos tobillos antes de iniciar su flexión plantar definitiva $\left(8^{\circ} \pm 3^{\circ}\right.$ y $9^{\circ} \pm 3^{\circ}$, para el tobillo de la pierna retrasada y adelantada, respectivamente). Es necesario considerar que, para la pierna adelantada, la dorsiflexión persiste hasta que comienza su flexión plantar al final de la fase de aceleración (ver figura 3(c)(f)). No se ha constatado flexión bilateral de las rodillas antes de iniciar su extensión definitiva (ver figura 3 (b), (e)), lo que indica que la contribución al desplazamiento hacia atrás del CM que ejercen las piernas se debe a la dorsiflexión de los tobillos antes de iniciar su flexión plantar.

Los datos expuestos son coincidentes con los reportados en otras publicaciones donde se constata la existencia de una dorsiflexión bilateral de los tobillos antes de iniciarse su flexión plantar definitiva (Brazil et al., 2017; Debaere et al., 2013; Guissard et al.,1992; Mero et al., 2006; Schrödter et al.,2017). Sin embargo, debemos cuestionar la asociación de este movimiento dorsiflesor de los tobillos a una actividad reactiva del ciclo de estiramiento acortamiento, como se sugiere en algunas de estas aportaciones, al menos desde un criterio puramente fisiológico o biomecánico, donde se busca tener un cierto grado de activación de los músculos propulsores cuando comienza el movimiento hacia adelante. En estas condiciones, probablemente la musculatura extensora no tenga el tiempo necesario para resistirse activamente al estiramiento previo a su posterior actividad concéntrica, un requisito imprescindible para obtener un cierto beneficio del ciclo estiramiento acortamiento. Es más probable que la dorsiflexión sea el efecto de la fuerza reactiva que ejerce el tronco+cabeza en su aceleración hacia delante y los músculos flexores plantares no consigan activarse para resistirse a esa fuerza reactiva que produce el movimiento hacia atrás de las piernas.

La contribución temprana de la cabeza+tronco al desplazamiento horizontal del CM se ha correlacionado positivamente con la velocidad horizontal al final de 
$0.312 \mathrm{~s}$ de la fase de aceleración $(\boldsymbol{r}=0.622, \mathrm{p}=0.003)$, lo que confirma su importancia como indicador de rendimiento de las salidas de tacos. Estos datos serían coherentes con las aportaciones de Schrödter et al. (2017) cuando hacen referencia a las correlaciones positivas entre velocidad de estiramiento dorsiflexor y la potencia desarrollada al final de la fase de aceleración. Efectivamente, una mayor contribución de la cabeza+tronco también supondría una mayor fuerza reactiva hacia atrás y, probablemente, una mayor velocidad de estiramiento de la musculatura durante la dorsiflexión. En este sentido, Slawinski et al. (2013) han relacionado el rendimiento de las salidas de tacos con la energía desarrollada por el tronco y la cabeza, confirmando la importancia que tiene la acción temprana de estos dos segmentos. La relevancia de la contribución temprana del tronco+cabeza al rendimiento también se ha constatado a partir de las aportaciones de Brazil et al. (2017), cuando señalan la importancia que tiene la musculatura extensora de la cadera para iniciar el movimiento desde parado. Como era de esperar, cuando finaliza la importante acción inicial del tronco + cabeza, su contribución al desplazamiento horizontal del CM se reduce a medida que aumenta su inclinación con respecto a la horizontal.

Tras el despegue, la pierna retrasada se desplaza hacia delante contribuyendo al desplazamiento horizontal del CM (8.1\% $\pm 1.0 \%$ al final el despegue del taco anterior). Además de esta importante contribución, la aceleración inicial de su masa produciría una cierta fuerza reactiva hacia atrás que pondría a la musculatura extensora de la pierna que está en contacto con el taco anterior en una actividad concéntrica más lenta, posibilitando así la mayor producción de fuerza muscular (Hill, 1938), lo que contribuiría al incremento en el registro de la fuerza horizontal resultante (figura 1(a)).

Como se ha indicado, los brazos mantienen una contribución horizontal negativa al desplazamiento horizontal del CM hasta el despegue del taco posterior, es decir, tienden a desplazar el CM hacia atrás. Este efecto negativo persistirá inevitablemente mientras que las manos estén en contacto con el suelo, aunque sería posible evitarlo tras su despegue. Tras el despegue del taco posterior, el brazo correspondiente al taco anterior comienza una cierta contribución positiva mientras que el brazo que corresponde al taco posterior sigue incrementando su contribución negativa (ver figura 1(e)(f)). Se puede concluir indicando que el resultado medio de la contribución de los dos brazos al desplazamiento horizontal del CM es muy reducido, lo que es coincidente con las aportaciones de Graham-Smith, Natera \& Saunders (2014) donde constatan una cierta contribución de los brazos al impulso vertical, mientras su efecto fue nulo para impulso horizontal.

\section{Conclusión final}

El proceso de cálculo y la metodología utilizada han permitido determinar la contribución al desplazamiento horizontal del CM de cinco grupos de segmentos. Constatándose la existencia de una secuencia temporal que comienza con la participación del tronco+cabeza, seguida de la contribución positiva de las piernas apoyadas en los tacos y de la pierna libre tras su despegue del taco posterior, mientras que la contribución de los brazos es irrelevante. Especial relevancia ha tenido la posibilidad de analizar por primera vez la contribución horizontal del tronco + cabeza en las salidas de velocidad. Así, su aceleración horizontal al inicio del movimiento provoca que las piernas reciban momentáneamente una fuerza horizontal compresiva que produce un cierto desplazamiento hacia atrás, lo que podría estar relacionada con la dorsiflexión de los tobillos y/ o flexión de las rodillas.

\section{Aplicaciones prácticas}

La importancia de la contribución del tronco + cabeza al desplazamiento horizontal del CM durante los primeros instantes de la fase de empuje, pone de manifiesto el papel que juega la musculatura extensora del tronco, actuando desde una posición inicial donde las caderas deben estar más altas que los hombros. La contribución de la pierna libre tras su despegue sugiere la importancia que adquiere la musculatura flexora de la cadera para acelerar la pierna hacia delante.

A partir de las consideraciones expuestas sobre el origen de la dorxiflesión de los tobillos y las dudas sobre los posibles beneficios de una actividad reactiva del ciclo de estiramiento acortamiento, probablemente la mejor solución sería evitar la dorsiflexión de los tobillos utilizando tacos más altos y más inclinados con respecto a la horizontal. Con esta propuesta, la musculatura extensora del tronco y las piernas podrían desarrollar más fuerza al estar en condiciones concéntricas más lentas, aunque no existiría la probable activación refleja de los músculos flexores plantares. Sin embargo, acerca de este problema existen ciertas controversias que deberían abordarse en investigaciones futuras. 


\section{Referencias}

Bezodis, N.E., Salo, A.I.T., Trewartha, G. (2010). Choice of sprint start performance measure affects the performance-based ranking within a group of sprinters: which is the most appropriate measure? Sports Biomechanics, 9, 258-269. Doi:10.1080/14763141.2010.538713.

Bezodis, N.E., Salo, A.I.T., Trewartha, G. (2015). Relationships between lower-limb kinematics and block phase performance in a cross section of sprinters. European Journal of Sport Science, 15, 118-24. Doi: 10.1080/ 17461391.2014 .928915$.

Bezodis, N.E., Willwacher, S., Salo, A.I.T. (2019). The biomechanics of the track and field sprint start: A narrative review, Sports Medicine, 49(9), 1345-1364. Doi10.1007/s40279-019-01138-1

Bezodis, N.E., Walton, S.P. y Nagahara, R. (2019). Understanding the track and field sprint start through a functional analysis of the external forcé features which contribute to higher levels of block phase performance, Journal of Sports Sciences, 37(5), 560-567, Doi: 10.1080/ 02640414.2018.1521713.

Brazil, A., Exell, T., Wilson, C., Willwacher, S., Bezodis, I. y Irwin, G. (2017). Lower limb joint kinetics in the starting blocks and first stance in athletic sprinting, Journal of Sports Sciences, 35(16), 1629-1635, Doi: 10.1080/ 02640414.2016 .1227465$.

Brazil, A., Exell, T., Wilson, C., Willwacher, S., Bezodis, I.N. y Irwin, G. (2018). Joint kinetic determinants of starting block performance in athletic sprinting. Journal of Sports Sciences, 36(14), 1656-1662, Doi: 10.1080/ 02640414.2017.1409608

de Leva, P. (1996). Adjustments to Zatsiorsky-Seluyanovs segment inertia parameters. Journal of Biomechanics, 29(9), 1223-1230, Doi: 10.1016/0021-9290(95)001786.

Debaere, S., Delecluse, C., Aerenhouts, D., Hagman, F. y Jonkers, I. (2013). From block clearance to sprintrunning: characteristics underlying an effective transition. Journal of Sports Sciences, 31(2), 137-149, Doi:10.1080/ 02640414.2012.722225.

Graham-Smith, P., Natera A. y Saunders, S. (2014). Contribution of the arms in the sprint start and their influence on force and velocity characteristics, En K. Sato, W. A. Sands, S. Mizuguchi (Eds.) 32 International Conference of Biomechanics in Sports (pp, 578-581). Johnson City, T.N., USA.

Guissard, N., Duchateau, J. y Hainaut, K. (1992). EMG and mechanical changes during sprint starts at different front block obliquities. Medicine \& Science in Sports \& Exercise, 24(11), 1257-1263.

Gutiérrez-Dávila, M., Dapena, J. y Campos, J. (2006). The effect of muscular pre-tensing on the sprint start, Journal of Applied Biomechanics, 22(3), 194-201, Doi:10.1123/ jab.22.3.194.

Gutiérrez-Dávila, M., Amaro, F.J., Garrido, J.M. y Rojas, F.J. (2014). An analysis of two styles of arm action in the vertical countermovement jump, Sports Biomechanics, 13(2), 135-143, Doi: 10.1080/14763141.2014.910832

Harland, M.J. y Steele, J,R. (1997). Biomechanics of the sprint start, Sports Medicine, 23(1), 11-20. Doi: 01121642/97/0001-0011/\$05.00/0.

Hill, A.V. (1938) The heat of shortening and dynamic constants of muscle. Proceedings of the Rohal Society Series B 126, 136-195.

Mero, A., Kuitunen, S., Harland, M., Kyröläinen, H. y Komi, P.V. (2006). Effects of muscle - tendon length on joint moment and power during sprint starts, Journal of Sports Sciences, 24(2), 165-173. Doi: 10.1080/ 02640410500131753

Otsuka, M., Shim, J.K., Kurihara, T., Yoshioka, S., Nokata, M. y Isaka, T. (2014). Effect of expertise on 3D force application during the starting block phase and subsequent steps in sprint running. Journal of Applied Biomechanics. 30(3), 390 400. Doi:10.1123/jab.2013-0017.

Schrödter, E., Brüggemann, G.P. y Willwacher, S. (2017). Is soleus muscletendon-unit behavior related to groundforce application during the sprint start?, International Journal of Sports Physiology and Performance, 12, 448-54. Doi:10.1123/ijspp.2015-0512

Slawinski, J., Bonnefoy, A., Ontanon, G., Leveque, J.M., Miller, C., Riquet, A., Chèze, L. y Dumaset, R. (2010). Segment-interaction in sprint start: analysis of 3D angular velocity and kinetic energy in elite sprinters. Journal of Biomechanics, 43, 1494-1502. Doi:10.1016/ j.jbiomech.2010.01.044

Slawinski, J., Dumas, R., Cheze, L., Ontanon, G., Miller, C. y Mazure-Bonnefoy, A. (2013). Effect of postural changes on 3D joint angular velocity during starting block phase. Journal of Sports Sciences, 31, 256-63. Doi: 10.1055/s-0032-1304587.

Willwacher, S., Herrmann, V., Heinrich, K., Funken, J., Strutzenberger, G., Goldmann, J.P., Braunstein, B., Brazil, A., Irwin, G., Potthast, W. y Brüggemann, G,P. (2016) Sprint Start Kinetics of Amputee and NonAmputee Sprinters. PLoS ONE, 11(11), e0166219, Doi:10.1371/journal.pone.0166219.

Wood, G. A. y Jennings, L. S. (1979). On the use of spline functions for data smoothing. Journal of Biomechanics, 12, 477-479, Doi:10.1016/S0021-9290(99)00083-4

Zatsiorsky, V. M., \& Seluyanov, N. V. (1983). The mass and inertial characteristics of the main segments of the human body. En H. Matsui \& K. Kobayashi (Eds.), Biomechanics VIII-B (pp. 1152-1159). Champaign, IL: Human Kinetics. 